\title{
Prognostic significance of peripheral blood absolute lymphocyte count and derived neutrophil to lymphocyte ratio in patients with newly diagnosed extranodal natural killer/T-cell lymphoma
}

This article was published in the following Dove Press journal:

Cancer Management and Research

\author{
Xiangxiang Zhou' ${ }^{\mathrm{l} *}$ \\ Xiaogang Sun ${ }^{1}$ * \\ Wenbo Zhao' \\ Xiaosheng Fang' \\ Xin Wang ${ }^{1,2}$
}

'Department of Hematology, Shandong Provincial Hospital Affiliated to Shandong University, Jinan, People's Republic of China; ${ }^{2}$ Department of Diagnostics, School of Medicine, Shandong University, Jinan, People's Republic of China

*These authors contributed equally to this work
Correspondence: Xin Wang

Department of Hematology, Shandong

Provincial Hospital Affiliated to Shandong

University, No.324, Jingwu Road, Jinan,

Shandong, 25002I, 193397, People's

Republic of China

Tel 8653168776358

Fax +865318706 II97

Email xinw@sdu.edu.cn
Background: Accumulating evidence suggested that tumor microenvironment and host immune system played important roles in determining the clinical course and outcome of human malignancies. The derived neutrophil to lymphocyte ratio (dNLR) and absolute lymphocyte count (ALC) were demonstrated to act as a prognostic factor in several malignancies. Nevertheless, the prognostic significance of them in extranodal natural killer/T-cell lymphoma (ENKTL) patients has never been explored.

Patients and methods: A total of 33 newly diagnosed patients with ENKTL were included in this study. Clinicopathological characteristics were collected and prognostic significance of dNLR and ALC were evaluated.

Results: Elevated dNLR and low ALC were both associated with poor survival rates. Patients with dNLR $\geq 3.6$ revealed significantly shorter overall survival (OS) $(P=0.001)$ and progression-free survival (PFS) $(P=0.008)$ than those with dNLR $<3$.6. Patients with ALC $<0.8 \times 10^{9} / \mathrm{L}$ had worse OS $(P=0.008)$ and PFS $(P<0.001)$ than those with ALC $\geq 0.8 \times 10^{9} / \mathrm{L}$. An independent significant association between low ALC and poor clinical outcome in multivariate analysis for OS (HR, 36.023; 95\% CI, 2.438-532.243; $P=0.009$ ) as well as PFS (HR, 7.698; 95\%CI, 1.573-37.679; $P=0.012$ ) was identified.

Conclusion: In this study, we validated for the first time the prognostic value of dNLR and ALC in ENKTL patients. Elevated dNLR and low ALC were both associated with aggressive tumor process and poor survival.ALC value at diagnosis represented an independent favorable prognostic factor for the clinical outcome of ENKTL patients.

Keywords: derived neutrophil to lymphocyte ratio, absolute lymphocyte count, extranodal NK/T-cell lymphoma, prognosis, progression-free survival, overall survival

\section{Introduction}

Extranodal natural killer/T-cell lymphoma (ENKTL) is a rare and highly aggressive lymphoma derived from mature NK- and T-cells, which accounts for less than $1 \%$ of non-Hodgkin lymphoma (NHL) in the Western world and up to $10 \%$ of NHL in Asia and South America. ${ }^{1}$ This disease is characterized by Epstein-Barr virus (EBV) infection and extranodal involvement, with 5-year overall survival (OS) rates of less than $50 \% .^{2-4}$ ENKTL can be pathologically divided into two types, nasal and non-nasal ENKTL. ${ }^{4}$ About $80 \%$ of ENKTL cases occur in the nose, nasopharynx, oropharynx, and parts of the upper aerodigestive tract, and about $20 \%$ of them occur 
in non-nasal sites, including skin, testis, and salivary glands. Patients with nasal or paranasal involvement are defined as nasal type. Non-nasal cases presented more adverse clinical features and poor survival outcome. ${ }^{5}$ At present, no standard treatment has been established due to the rarity of this disease. Although L-asparaginase-based chemotherapy regimens and radiotherapy have brought improvement in the treatment strategy of ENKTL, the prognosis of these patients is still unsatisfactory. ${ }^{1,6,7}$

The International Prognostic Index (IPI), including age, Ann Arbor stage, serum lactate dehydrogenase (LDH), performance status (PS), and number of extranodal sites, is a useful scoring model for predicting the prognosis of NHL. ${ }^{8}$ With the abandonment of anthracycline-based regimens and appearance of L-asparaginase-containing regimens, a new prognostic model, prognostic index for NK/T cell lymphoma (PINK), was established. ${ }^{9}$ The PINK score consists of four factors, including age, stage, distant lymph-node involvement, and non-nasal type. The prognostic significance of IPI and PINK in ENKTL patients have been verified in many studies.

In recent years, several studies have suggested that tumor microenvironment and host immune system played important roles in the pathogenesis and clinical outcome of human malignancies. Accumulating evidence suggested that the pre-treatment derived neutrophil to lymphocyte ratio (dNLR) was demonstrated to act as a significant prognostic factor in a variety of solid tumors, including non-small cell lung cancer, esophageal cancer, gastric cancer, breast cancer, and melanoma. ${ }^{10-14}$ Nevertheless, the role of dNLR in hematological malignancies was rarely reported. ${ }^{15}$

Lymphocytes play a crucial role in the tumor-related immune response. Elevated levels of absolute lymphocyte count (ALC) were demonstrated to be associated with favorable prognosis in several types of solid tumors, such as gastric cancer, bladder cancer and ovarian cancer. ${ }^{16-19}$ As a surrogate of host immunity impairment, ALC was reported to be a prognostic indicator in hematological malignancies, including multiple myeloma (MM), primary central nervous system lymphoma (PCNSL), and anaplastic large cell lymphoma (ALCL). ${ }^{20-22}$ In addition, ALC was also incorporated into the International Prognostic Score (IPS) of Hodgkin lymphoma (HL). ${ }^{23,24}$

However, the significance of dNLR and ALC in the tumor progression and treatment response in patients with ENKTL is still unclear. In the present study, we evaluated the prognostic value of dNLR and ALC in newly diagnosed ENKTL patients.

\section{Patients and methods}

\section{Study population}

We conducted a retrospective study of a cohort of 33 patients with newly diagnosed ENKTL from February 2010 to June 2018 at Shandong Provincial Hospital affiliated to Shandong University. All diagnoses were based on the criteria of the WHO 2016 Classifications of the Tumors and Hematopoietic and Lymphoid Tissues. ${ }^{25}$ Inclusion criteria were histopathologically confirmed of ENKTL, and pre-treatment values of white blood cell (WBC) count, ALC, absolute neutrophil count (ANC), and other clinical data. This study was approved by the Medical Ethical Committee of Shandong Provincial Hospital affiliated to Shandong University. All data of the recruited patients were obtained with written informed consent in accordance with the Declaration of Helsinki.

\section{Data gathering}

The pretreatment clinical-pathological data, such as age, gender, Ann-Arbor stage, B symptoms, Eastern Cooperative Oncology Group (ECOG) performance status (PS) score, serum LDH levels, bone marrow involvement, $\beta 2$-microglobulin ( $\beta 2-\mathrm{MG})$, blood EBV-DNA level, WBC, ANC, ALC, PINK score, and IPI score were collected.

\section{Calculation of derived neutrophil/ lymphocyte ratio (dNLR)}

The WBC and ANC were obtained at patient enrollment. The dNLR was calculated by a formula as $[\mathrm{dNLR}=\mathrm{ANC} /$ (WBC-ANC)], which was previously verified to have predictive power in cancer patients. ${ }^{26}$

\section{Treatment}

The therapeutic strategies of the included patients consist of chemotherapy alone, radiotherapy alone and combined chemoradiotherapy. The chemotherapy regimens included P-CHOP (pegaspargase, cyclophosphamide, doxorubicin, vincristine, and prednisone), P-CHOPE (pegaspargase, CHOP, and etoposide), L-EPOCH (L-asparaginase, etoposide, prednisone, vincristine, cyclophosphamide, and doxorubicin), SMILE (dexamethasone, methotrexate, ifosfamide, pegaspargase, and etoposide), GELOX (gemcitabine, pegaspargase, and oxaliplatin) and P-GemOX (pegaspargase, gemcitabine, and oxaliplatin). Radiotherapy used was no more than 56 Gy (range, 42 to $56 \mathrm{~Gy}$ ). Response to the treatment includes complete remission (CR) or partial remission (PR), no 
response to treatment includes stable disease (SD) or progressive disease (PD).

\section{Statistic analysis}

OS was defined as interval from the date of diagnosis to the date of last follow-up or death. Progression-free survival (PFS) was calculated as interval from the date of diagnosis to date of disease progression. The differences of the quantitative variables were compared by Mann-Whitney U test or Student's $t$-test. Fisher exact test was used for categorical variables. The optimal cutoff points of dNLR and ALC were determined by receiver operating characteristics curve (ROC) and area under curve (AUC). Survival analyses were performed using the Kaplan-Meier method and the log-rank test was used to calculate for significant differences. Univariate and multivariate analyses were performed using the Cox proportional-hazards regression model. Statistical analyses were performed by SPSS software for Windows ver. 20.0 (SPSS Inc, Chicago, IL, USA). $P$-value of $<0.05$ was considered statistically significant.

\section{Results}

\section{Clinical characteristics of patients}

A total of 33 patients with pathological diagnosis of ENKTL were included in this study, including 26 (78.8\%) male patients and $7(21.1 \%)$ female patients. The median age at diagnosis was 50 years (range, 13-75 years), and $6(18.2 \%)$ of them were more than 60 years old. About $60.6 \%(n=20)$ of them presented B symptoms. The number of patients with stage I/II and III/IV were 13 $(39.4 \%)$ and $20(60.6 \%)$, respectively. The majority of them $(78.8 \%, n=26)$ had good performance status (ECOG PS score 0/1). Nineteen (58\%) of the included patients revealed elevated $\beta 2-\mathrm{MG}$ level and 16 (48.5\%) patients presented high IPI score (IPI $\geq 2$ ). patients were treated with chemotherapy alone $(51.5 \%, \mathrm{n}=17)$, radiotherapy alone $(33.3 \%, n=11)$ or combined chemoradiotherapy $(15.2 \%, n=5)$. One patient received salvage allogeneic hematopoietic stem cell transplantation (HSCT). Among them, $15(45.5 \%)$ exhibited a response to the first-line therapy. The baseline clinical characteristics are described in Table 1.

\section{The association between clinicopathologic variables and $d N L R, A L C$}

We next assessed the association between clinicopathologic variables and dNLR as well as ALC in the included
ENKTL patients. Among them, the median value of dNLR was 1.79 (range, 0.427-7.6). 39.4\% $(n=13)$ patients were diagnosed at stage I/II with the mean dNLR of 1.801 \pm 0.832 , versus $60.6 \%(\mathrm{n}=20)$ at stage III/IV with the mean dNLR of $2.834 \pm 1.962(P=0.047)$. In addition, the dNLR levels were significantly higher in ENKTL patients with elevated serum LDH $(P=0.026)$, distant lymph-node involvement $(P=0.013)$, high $\beta 2-\mathrm{MG}(P=0.035)$, and high IPI score $(P=0.044$, Table 1$)$. Low ALC levels were significantly associated with high ECOG PS score $(P=0.002)$, elevated serum LDH $(P=0.01)$, distant lymph-node involvement $(P=0.026)$, elevated $\beta 2-\mathrm{MG}(P=0.029)$, high IPI score $(P=0.024)$, and increased blood EBV DNA levels $(P=0.011$, Table 1).

\section{Correlation between $\mathrm{dNLR}, \mathrm{ALC}$, and clinical outcomes}

The ROC analysis was performed to determine the specificity and sensitivity of $\mathrm{dNLR}$ and ALC, respectively. The most discriminative cutoff value of dNLR was 3.6, with an AUC value of 0.714 (95\% confidence interval [CI], $0.524-0.904, P=0.043$, Figure 1A). The most discriminative cutoff value of ALC was $0.8 \times 10^{9} / \mathrm{L}$, with an $\mathrm{AUC}$ value of 0.738 (95\% CI, 0.523-0.953, $P=0.025$, Figure 1B). Based on the above results, we selected dNLR of 3.6 and ALC of $0.8 \times 10^{9} / \mathrm{L}$ as the optimal cutoff points for survival analysis in the entire cohort.

Clinical indicators according to the dNLR and ALC at diagnosis are shown in Table 2. Patients with dNLR of $\geq 3.6$ presented adverse pre-treatment factors, including higher ECOG PS score $(P=0.004)$, elevated serum LDH level $(P<0.001)$, increased $\beta 2-\mathrm{MG}(P=0.008)$, and high IPI score $(P=0.018$, Table 2$)$. In addition, patients with dNLR $\geq 3.6$ showed worse response to treatment compared to those with dNLR $<3.6(P=0.049$, Table 2$)$. Patients with ALC of $<0.8 \times 10^{9} / \mathrm{L}$ presented high ECOG PS score $(P=0.009)$, elevated serum LDH level $(P=0.001)$, increased $\beta 2-\mathrm{MG}$ level $(P=0.02)$, elevated blood EBV DNA level $(P=0.019)$, and distant lymph-node involvement $(P=0.047$, Table 2). ENKTL patients with ALC $<0.8 \times 10^{9} / \mathrm{L}$ revealed more inferior treatment response compared to those with ALC $\geq 0.8 \times 10^{9} / \mathrm{L}(P=0.021$, Table 2$)$.

\section{Survival analysis of $d N L R$ and ALC}

Survival analysis revealed that ENKTL patients with high dNLR $(\geq 3.6)$ revealed significantly shorter OS $(P=0.001)$ and PFS $(P=0.008)$ than those with low 
Table I Correlation between dNLR and clinical characteristics of ENKTL patients

\begin{tabular}{|c|c|c|c|c|c|}
\hline & \multirow[t]{2}{*}{ Total (\%) } & \multicolumn{2}{|l|}{ dNLR } & \multicolumn{2}{|l|}{ ALC } \\
\hline & & Mean \pm SD & $P$-value & Mean \pm SD & $P$-value \\
\hline $\begin{array}{l}\text { Gender } \\
\text { Male } \\
\text { Female }\end{array}$ & $\begin{array}{l}33 \\
26(78.8 \%) \\
7(21.2 \%)\end{array}$ & $\begin{array}{l}2.658 \pm 1.804 \\
1.570 \pm 0.584\end{array}$ & 0.129 & $\begin{array}{l}1.264 \pm 0.616 \\
1.109 \pm 0.690\end{array}$ & 0.602 \\
\hline $\begin{array}{l}\text { Age (year) } \\
\quad<60 \\
\geq 60\end{array}$ & $\begin{array}{l}27(81.8 \%) \\
6(18.2 \%)\end{array}$ & $\begin{array}{l}2.503 \pm 1.787 \\
2.084 \pm 1.095\end{array}$ & 0.588 & $\begin{array}{l}\text { I. } 208 \pm 0.602 \\
\text { I. } 333 \pm 0.774\end{array}$ & 0.664 \\
\hline $\begin{array}{l}\text { Ann Arbor Stage } \\
\text { I/II } \\
\text { III/IV }\end{array}$ & $\begin{array}{l}13(39.4 \%) \\
20(60.6 \%)\end{array}$ & $\begin{array}{l}1.80 I \pm 0.832 \\
2.834 \pm 1.962\end{array}$ & 0.047 & $\begin{array}{l}1.410 \pm 0.539 \\
I .115 \pm 0.66 \mid\end{array}$ & 0.188 \\
\hline $\begin{array}{l}\text { B symptoms } \\
\text { Absence } \\
\text { Presence }\end{array}$ & $\begin{array}{l}13(39.4 \%) \\
20(60.6 \%)\end{array}$ & $\begin{array}{l}2.018 \pm 0.807 \\
2.693 \pm 2.033\end{array}$ & 0.194 & $\begin{array}{l}I .22 I \pm 0.523 \\
I .238 \pm 0.693\end{array}$ & 0.941 \\
\hline $\begin{array}{l}\text { ECOG PS score } \\
0 \text { or } 1 \\
\geq 2\end{array}$ & $\begin{array}{l}26(78.8 \%) \\
7(21.2 \%)\end{array}$ & $\begin{array}{l}1.942 \pm 0.925 \\
4.612 \pm 2.556\end{array}$ & 0.050 & $\begin{array}{l}I .38 I \pm 0.550 \\
0.555 \pm 0.50 I\end{array}$ & 0.002 \\
\hline $\begin{array}{l}\text { Serum LDH } \\
\text { Normal } \\
\text { Increased }<3 \times \mathrm{NL} \\
\text { Increased } \geq 3 \times \mathrm{NL}\end{array}$ & $\begin{array}{l}18(54.5 \%) \\
9(27.3 \%) \\
6(18.3 \%)\end{array}$ & $\begin{array}{l}2.235 \pm 1.272 \\
1.763 \pm 1.243 \\
3.999 \pm 2.474\end{array}$ & 0.026 & $\begin{array}{l}I .478 \pm 0.476 \\
I .130 \pm 0.46 \mid \\
0.642 \pm 0.854\end{array}$ & 0.01 \\
\hline $\begin{array}{l}\text { Extranodal site } \\
\quad 0 \text { or } 1 \\
\geq 2\end{array}$ & $\begin{array}{l}21(63.6 \%) \\
12(36.4 \%)\end{array}$ & $\begin{array}{l}2.233 \pm 1.285 \\
2.768 \pm 2.230\end{array}$ & 0.458 & $\begin{array}{l}1.331 \pm 0.584 \\
1.057 \pm 0.679\end{array}$ & 0.231 \\
\hline $\begin{array}{l}\text { Distant lympho node } \\
\text { Not involved } \\
\text { Involved }\end{array}$ & $\begin{array}{l}13(39.4 \%) \\
20(60.6 \%)\end{array}$ & $\begin{array}{l}1.651 \pm 0.745 \\
2.932 \pm 1.919\end{array}$ & 0.013 & $\begin{array}{l}1.525 \pm 0.460 \\
1.040 \pm 0.652\end{array}$ & 0.026 \\
\hline $\begin{array}{l}\text { BM involvement } \\
\text { Absence } \\
\text { Presence }\end{array}$ & $\begin{array}{l}31(93.9 \%) \\
2(6.1 \%)\end{array}$ & $\begin{array}{l}2.348 \pm 1.55 \mid \\
3.648 \pm 3.824\end{array}$ & 0.715 & $\begin{array}{l}1.278 \pm 0.612 \\
0.495 \pm 0.318\end{array}$ & 0.085 \\
\hline $\begin{array}{l}\text { B2-microglobulin } \\
\quad<2.8 \mathrm{mg} / \mathrm{L} \\
\geq 2.8 \mathrm{mg} / \mathrm{L}\end{array}$ & $\begin{array}{l}19(58 \%) \\
14(42 \%)\end{array}$ & $\begin{array}{l}1.825 \pm 0.775 \\
3.245 \pm 2.198\end{array}$ & 0.035 & $\begin{array}{l}1.45 I \pm 0.429 \\
0.933 \pm 0.734\end{array}$ & 0.029 \\
\hline $\begin{array}{l}\text { Blood EBV DNA } \\
<5,000 \text { copies } / \mathrm{mL} \\
\geq 5,000 \text { copies } / \mathrm{mL}\end{array}$ & $\begin{array}{l}19(57.6 \%) \\
14(42.4 \%)\end{array}$ & $\begin{array}{l}1.991 \pm 0.942 \\
3.019 \pm 2.243\end{array}$ & 0.126 & $\begin{array}{l}1.462 \pm 0.527 \\
0.917 \pm 0.625\end{array}$ & 0.011 \\
\hline $\begin{array}{l}\text { Treatment } \\
\text { RT alone } \\
\text { CT alone } \\
\text { Combined RT and CT }\end{array}$ & $\begin{array}{l}11(33.3 \%) \\
17(51.5 \%) \\
5(15.2 \%)\end{array}$ & $\begin{array}{l}2.418 \pm 1.877 \\
2.533 \pm 1.786 \\
2.087 \pm 0.849\end{array}$ & 0.879 & $\begin{array}{l}\text { I. } 394 \pm 0.522 \\
\text { I. } 109 \pm 0.678 \\
\text { I. } 286 \pm 0.678\end{array}$ & 0.504 \\
\hline $\begin{array}{l}\text { Response } \\
\quad \text { No response (SD, PD) } \\
\text { Response (CR, PR) }\end{array}$ & $\begin{array}{l}18(54.5 \%) \\
\text { I5 (45.5\%) }\end{array}$ & $\begin{array}{l}2.819 \pm 2.075 \\
1.957 \pm 0.87 \mid\end{array}$ & 0.123 & $\begin{array}{l}1.141 \pm 0.752 \\
1.339 \pm 0.425\end{array}$ & 0.349 \\
\hline
\end{tabular}

(Continued) 
Table I (Continued).

\begin{tabular}{|c|c|c|c|c|c|}
\hline & \multirow[t]{2}{*}{ Total (\%) } & \multicolumn{2}{|l|}{ dNLR } & \multicolumn{2}{|l|}{ ALC } \\
\hline & & Mean \pm SD & $P$-value & Mean \pm SD & $P$-value \\
\hline $\begin{array}{l}\text { PINK score } \\
0 \text { or } 1 \\
\geq 2\end{array}$ & $\begin{array}{l}17(51.5 \%) \\
16(48.5 \%)\end{array}$ & $\begin{array}{l}2.088 \pm 1.338 \\
2.788 \pm 1.953\end{array}$ & 0.236 & $\begin{array}{l}1.394 \pm 0.524 \\
1.058 \pm 0.690\end{array}$ & 0.125 \\
\hline $\begin{array}{l}\text { IPI Score } \\
0 \text { or I } \\
\geq 2\end{array}$ & $\begin{array}{l}17(51.5 \%) \\
16(48.5 \%)\end{array}$ & $\begin{array}{l}1.837 \pm 0.787 \\
3.055 \pm 2.126\end{array}$ & 0.044 & $\begin{array}{l}|.465 \pm 0.57| \\
0.923 \pm 0.597\end{array}$ & 0.024 \\
\hline
\end{tabular}

Note: Bold signifies $P<0.05$.

Abbreviations: ALC, absolute lymphocyte count; dNLR, derived neutrophil to lymphocyte ratio; ECOG PS, Eastern Cooperative Oncology Group performance status; ENKTL, extranodal natural killer/T-cell lymphoma; LDH, lactate dehydrogenase; BM, bone marrow; EBV, Epstein-Barr virus; RT, radiotherapy; CT, chemotherapy; CR, complete remission; PR, partial remission; SD, stable disease; PD, progressive disease; PINK, prognostic index for NK/T cell lymphoma; IPI, international prognostic index; ULN, upper limit of normal.
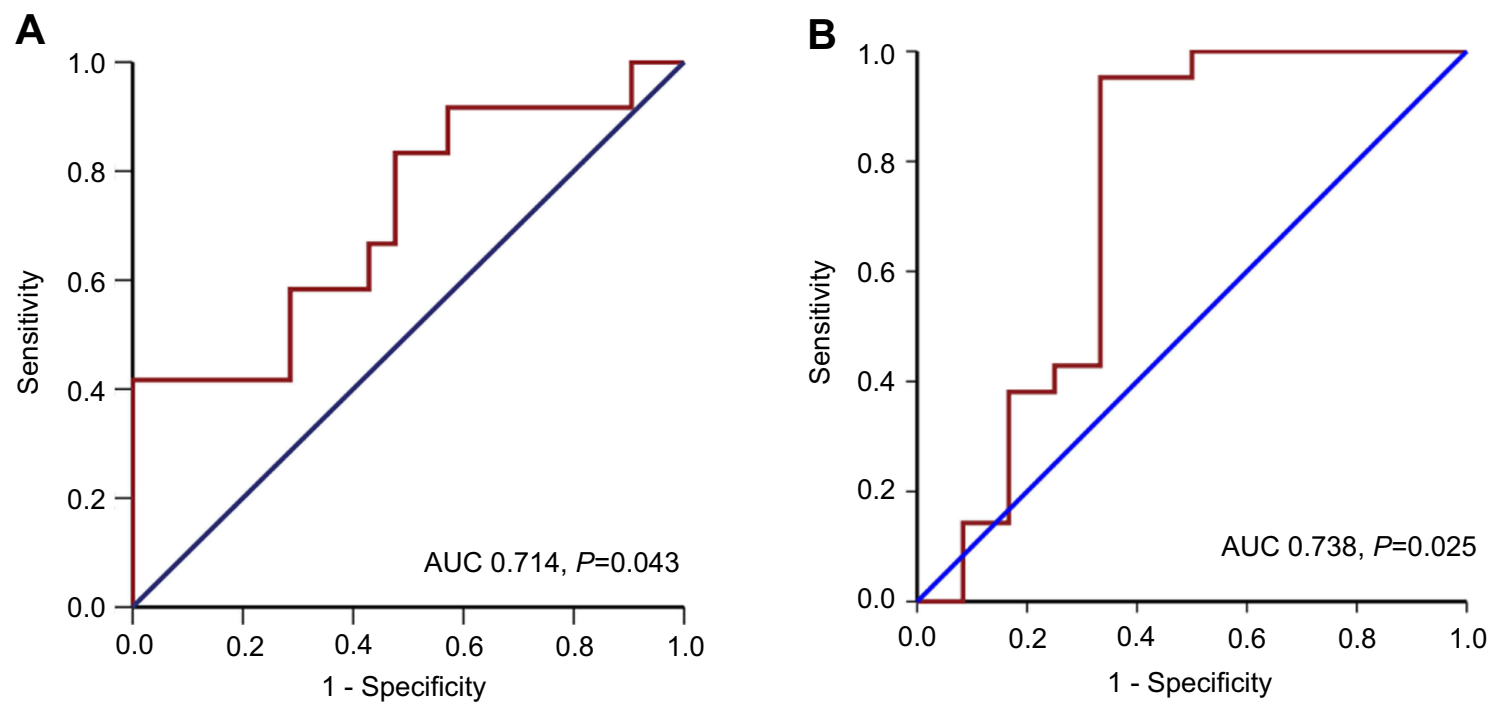

Figure I ROC curves for dNLR (A) and ALC (B) at diagnosis.

Abbreviations: ALC, absolute lymphocyte count; AUC, area under the curve; dNLR, derived neutrophil to lymphocyte ratio; ROC, receiver operating characteristic.

dNLR (<3.6, Figures 2A and 3A). Patients with an ALC of $<0.8 \times 10^{9} / \mathrm{L}$ had worse $\mathrm{OS}(P<0.001)$ and $\mathrm{PFS}$ $(P<0.001)$ than those with an $\mathrm{ALC}$ of $\geq 0.8 \times 10^{9} / \mathrm{L}$ (Figures $2 \mathrm{~B}$ and $3 \mathrm{~B}$ ).

The prognostic value of dNLR was further evaluated in accordance with stage. In advanced stage (stage III/IV) ENKTL patients, dNLR $\geq 3.6$ signified shorter survival for both OS $(P=0.048)$ and PFS $(P=0.026$, Figure $4 \mathrm{~A}$ and $\mathrm{B})$, while in early stage (stage $\mathrm{I} / \mathrm{II}$ ) patients, $\mathrm{dNLR} \geq 3.6$ presented no significant difference in the OS and PFS of patients. We further analyzed the effect of ALC on the survival of ENKTL patients according to stage. Advanced stage (stage III/IV) patients with ALC $<0.8 \times 10^{9} / \mathrm{L}$ exhibited significantly shorter OS $(P=0.002)$ and PFS $(P=0.001$, Figure $4 \mathrm{C}$ and D). Nevertheless, no significant difference of survival time (OS,
PFS) was found between low ALC group (ALC $<0.8 \times 10^{9} / \mathrm{L}$ ) and high ALC group (ALC $\geq 0.8 \times 10^{9} / \mathrm{L}$ ) in ENKTL patients with early stage (stage I/II).

\section{Analysis of prognostic indicators}

We next explored the correlation between clinical characteristics and survival of ENKTL patients using univariate and multivariate analyses. Univariate Cox proportional analysis revealed that high ECOG PS score $(\geq 2$; HR, 4.865; 95\%CI, 1.477-16.030; $P=0.009$ ), elevated $\beta 2-\mathrm{MG}$ ( $\geq 2.8 \mathrm{mg} / \mathrm{L}$; HR, 4.795; 95\%CI, 1.223-18.809; $P=0.025$ ), high IPI score $(\geq 2$; HR, 6.332; 95\%CI, 1.379-29.074; $P=0.018)$, low ALC $\left(<0.8 \times 10^{9} / \mathrm{L} ; \mathrm{HR}, 11.251 ; 95 \% \mathrm{CI}\right.$, 2.946-42.968; $P=0.0004)$ and elevated dNLR ( $>3.6$; HR, 5.938; 95\%CI, 1.790-19.704; $P=0.004)$ were correlated 
Table 2 Association between clinical variables and dNLR, ALC

\begin{tabular}{|c|c|c|c|c|c|c|c|}
\hline Variables & $\begin{array}{l}\text { Total } \\
(n=33)\end{array}$ & $\begin{array}{l}\mathrm{dNLR} \geq 3.6 \\
(n=5)\end{array}$ & $\begin{array}{l}\text { dNLR<3.6 } \\
(n=28)\end{array}$ & $P$-value & $\begin{array}{l}A L C \geq 0.8 \times 10^{9} / L \\
(n=24)\end{array}$ & $\begin{array}{l}\mathrm{ALC}<0.8 \times 10^{9} / \mathrm{L} \\
\mathrm{n}=9\end{array}$ & $P$-value \\
\hline $\begin{array}{c}\text { Gender } \\
\text { Male } \\
\text { Female }\end{array}$ & $\begin{array}{l}26 \\
7\end{array}$ & $\begin{array}{l}5(100.0 \%) \\
0(0.0 \%)\end{array}$ & $\begin{array}{l}21(75.0 \%) \\
7(25.0 \%)\end{array}$ & 0.559 & $\begin{array}{l}20(83.3 \%) \\
4(16.7 \%)\end{array}$ & $\begin{array}{l}6(66.7 \%) \\
3(33.3 \%)\end{array}$ & 0.358 \\
\hline $\begin{array}{l}\text { Age (year) } \\
\quad<60 \\
\geq 60\end{array}$ & $\begin{array}{l}27 \\
6\end{array}$ & $\begin{array}{l}4(80.0 \%) \\
\text { I (20.0\%) }\end{array}$ & $\begin{array}{l}23(82.1 \%) \\
5(17.9 \%)\end{array}$ & I & $\begin{array}{l}20(83.3 \%) \\
4(16.7 \%)\end{array}$ & $\begin{array}{l}7(77.8 \%) \\
2(22.2 \%)\end{array}$ & I \\
\hline $\begin{array}{l}\text { Ann Arbor Stage } \\
\text { I/II } \\
\text { III/IV }\end{array}$ & $\begin{array}{l}13 \\
20\end{array}$ & $\begin{array}{l}0(0.0 \%) \\
5(100.0 \%)\end{array}$ & $\begin{array}{l}13(46.4 \%) \\
15(53.6 \%)\end{array}$ & 0.131 & $\begin{array}{l}\text { II (45.8\%) } \\
\text { I } 3(54.2 \%)\end{array}$ & $\begin{array}{l}2(22.2 \%) \\
7(77.8 \%)\end{array}$ & 0.263 \\
\hline $\begin{array}{l}\text { B symptoms } \\
\text { Absence } \\
\text { Presence }\end{array}$ & $\begin{array}{l}13 \\
20\end{array}$ & $\begin{array}{l}0(0.0 \%) \\
5(100.0 \%)\end{array}$ & $\begin{array}{l}13(46.4 \%) \\
15(53.6 \%)\end{array}$ & 0.131 & $\begin{array}{l}10(41.7 \%) \\
14(58.3 \%)\end{array}$ & $\begin{array}{l}3(33.3 \%) \\
6(66.7 \%)\end{array}$ & I \\
\hline $\begin{array}{l}\text { ECOG PS score } \\
0 \text { or } 1 \\
\geq 2\end{array}$ & $\begin{array}{l}26 \\
7\end{array}$ & $\begin{array}{l}\text { I (20.0\%) } \\
4(80.0 \%)\end{array}$ & $\begin{array}{l}25(89.3 \%) \\
3(10.7 \%)\end{array}$ & 0.004 & $\begin{array}{l}22(91.7 \%) \\
2(8.3 \%)\end{array}$ & $\begin{array}{l}4(44.4 \%) \\
5(55.6 \%)\end{array}$ & 0.009 \\
\hline $\begin{array}{l}\text { Serum LDH } \\
\text { Normal } \\
\text { Increased }<3 \times \text { ULN } \\
\text { Increased } \geq 3 \times \text { ULN }\end{array}$ & $\begin{array}{l}18 \\
9 \\
6\end{array}$ & $\begin{array}{l}\text { I }(20.0 \%) \\
0(0.0 \%) \\
4(80.0 \%)\end{array}$ & $\begin{array}{l}17(60.7 \%) \\
9(32.1 \%) \\
2(7.2 \%)\end{array}$ & $<0.001$ & $\begin{array}{l}\text { I7 (70.8\%) } \\
6(25.0 \%) \\
\text { I (4.2\%) }\end{array}$ & $\begin{array}{l}\text { I }(11.1 \%) \\
3(33.3 \%) \\
5(55.6 \%)\end{array}$ & 0.001 \\
\hline $\begin{array}{l}\text { Distant lympho node } \\
\text { Not involved } \\
\text { Involved }\end{array}$ & $\begin{array}{l}13 \\
20\end{array}$ & $\begin{array}{l}0(0.0 \%) \\
5(100.0 \%)\end{array}$ & $\begin{array}{l}13(46.4 \%) \\
15(53.6 \%)\end{array}$ & 0.131 & $\begin{array}{l}\text { I3 (54.2\%) } \\
\text { II (45.8\%) }\end{array}$ & $\begin{array}{l}1(11.1 \%) \\
8(88.9 \%)\end{array}$ & 0.047 \\
\hline $\begin{array}{l}\text { BM involvement } \\
\text { Absence } \\
\text { Presence }\end{array}$ & $\begin{array}{l}31 \\
2\end{array}$ & $\begin{array}{l}4(80.0 \%) \\
\text { I (20.0\%) }\end{array}$ & $\begin{array}{l}27(96.4 \%) \\
I(3.6 \%)\end{array}$ & 0.284 & $\begin{array}{l}24(100.0 \%) \\
0(0.0 \%)\end{array}$ & $\begin{array}{l}7(77.8 \%) \\
2(22.2 \%)\end{array}$ & 0.068 \\
\hline $\begin{array}{l}\text { B2-microglobulin } \\
\quad<2.8 \mathrm{mg} / \mathrm{L} \\
\geq 2.8 \mathrm{mg} / \mathrm{L}\end{array}$ & $\begin{array}{l}19 \\
14\end{array}$ & $\begin{array}{l}0(0.0 \%) \\
5(100.0 \%)\end{array}$ & $\begin{array}{l}19(67.9 \%) \\
9(32.1 \%)\end{array}$ & 0.008 & $\begin{array}{l}18(75.0 \%) \\
6(25.0 \%)\end{array}$ & $\begin{array}{l}\text { I (II.1\%) } \\
8(88.9 \%)\end{array}$ & 0.02 \\
\hline $\begin{array}{l}\text { Blood EBV DNA } \\
\quad<5,000 \text { copies } / \mathrm{mL} \\
\geq 5,000 \text { copies } / \mathrm{mL}\end{array}$ & $\begin{array}{l}19 \\
14\end{array}$ & $\begin{array}{l}2(40.0 \%) \\
3(60.0 \%)\end{array}$ & $\begin{array}{l}17(60.7 \%) \\
11(39.3 \%)\end{array}$ & 0.628 & $\begin{array}{l}\text { I7 (70.8\%) } \\
7(29.2 \%)\end{array}$ & $\begin{array}{l}2(22.2 \%) \\
7(77.8 \%)\end{array}$ & 0.019 \\
\hline $\begin{array}{l}\text { Treatment } \\
\text { RT alone } \\
\text { CT alone } \\
\text { Combined RT and CT }\end{array}$ & $\begin{array}{l}11 \\
17 \\
5\end{array}$ & $\begin{array}{l}\text { I (20.0\%) } \\
4(80.0 \%) \\
0(0.00 \%)\end{array}$ & $\begin{array}{l}10(35.7 \%) \\
13(46.4 \%) \\
5(17.9 \%)\end{array}$ & 0.344 & $\begin{array}{l}10(41.7 \%) \\
10(41.7 \%) \\
4(16.7)\end{array}$ & $\begin{array}{l}\text { I (II.I\%) } \\
7(77.8 \%) \\
\text { I (II.I\%) }\end{array}$ & 0.163 \\
\hline $\begin{array}{l}\text { Response } \\
\text { No response (SD, PD) } \\
\text { Response (CR, PR) }\end{array}$ & $\begin{array}{l}18 \\
15\end{array}$ & $\begin{array}{l}5(100.0 \%) \\
0(0.0 \%)\end{array}$ & $\begin{array}{l}13(46.4 \%) \\
15(53.6 \%)\end{array}$ & 0.049 & $\begin{array}{l}10(41.7 \%) \\
14(58.3 \%)\end{array}$ & $\begin{array}{l}8 \text { (88.9\%) } \\
\text { I (II.I\%) }\end{array}$ & 0.021 \\
\hline $\begin{array}{l}\text { PINK } \\
0 \text { or I } \\
\geq 2\end{array}$ & $\begin{array}{l}17 \\
16\end{array}$ & $\begin{array}{l}\text { I (20.0\%) } \\
4(80.0 \%)\end{array}$ & $\begin{array}{l}16(57.1 \%) \\
12(42.9 \%)\end{array}$ & 0.175 & $\begin{array}{l}14(58.3 \%) \\
10(41.7 \%)\end{array}$ & $\begin{array}{l}3 \text { (33.3\%) } \\
6 \text { (66.7\%) }\end{array}$ & 0.269 \\
\hline
\end{tabular}

(Continued) 
Table 2 (Continued).

\begin{tabular}{|c|c|c|c|c|c|c|c|}
\hline Variables & $\begin{array}{l}\text { Total } \\
(n=33)\end{array}$ & $\begin{array}{l}d N L R \geq 3.6 \\
(n=5)\end{array}$ & $\begin{array}{l}\text { dNLR<3.6 } \\
(n=28)\end{array}$ & $P$-value & $\begin{array}{l}A L C \geq 0.8 \times 10^{9} / L \\
(n=24)\end{array}$ & $\begin{array}{l}\text { ALC }<0.8 \times 10^{9} / L \\
n=9\end{array}$ & $P$-value \\
\hline IPI Score & & & & 0.018 & & & 0.057 \\
\hline 0 or 1 & 17 & $0(0.0 \%)$ & $17(60.7 \%)$ & & 15 (62.5\%) & 2 (22.2\%) & \\
\hline$\geq 2$ & 16 & $5(100.0 \%)$ & II (39.3\%) & & 9 (37.5\%) & 7 (77.8\%) & \\
\hline
\end{tabular}

Note: Bold signifies $P<0.05$.

Abbreviations: ALC, absolute lymphocyte count; dNLR, derived neutrophil to lymphocyte ratio; ALC, absolute lymphocyte count; ECOG PS, Eastern Cooperative Oncology Group performance status; LDH, lactate dehydrogenase; BM, bone marrow; EBV, epstein-barr virus; RT, radiotherapy; CT, chemotherapy; CR, complete remission; PR, partial remission; SD, stable disease; PD, progressive disease; PINK, prognostic index for NK/T cell lymphoma; IPI, international prognostic index; ULN, upper limit of normal.
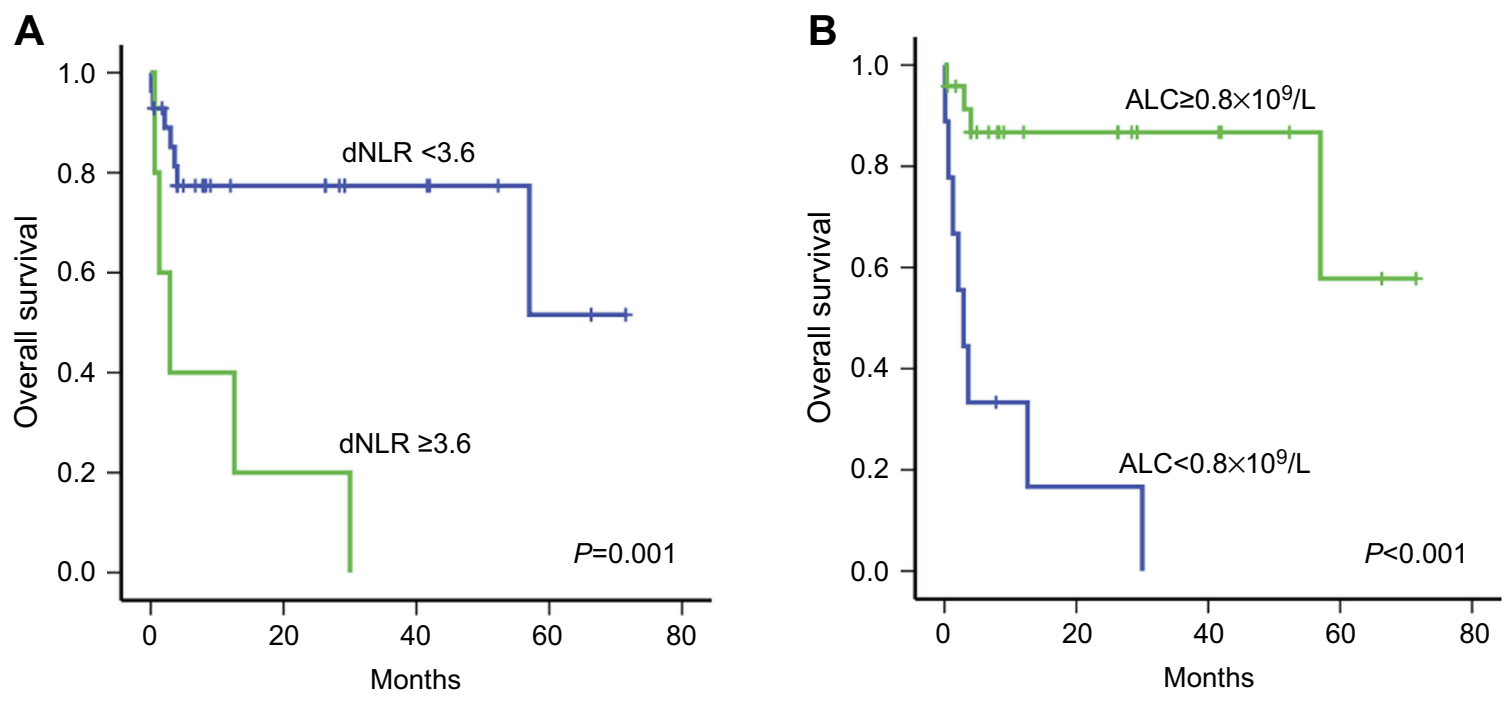

Figure 2 Kaplan-Meier curve of overall survival according to dNLR (A) and ALC (B) at diagnosis in patients with ENKTL.

Abbreviations: ALC, absolute lymphocyte count; AUC, area under the curve; dNLR, derived neutrophil to lymphocyte ratio; ENKTL, extranodal natural killer/T-cell lymphoma.
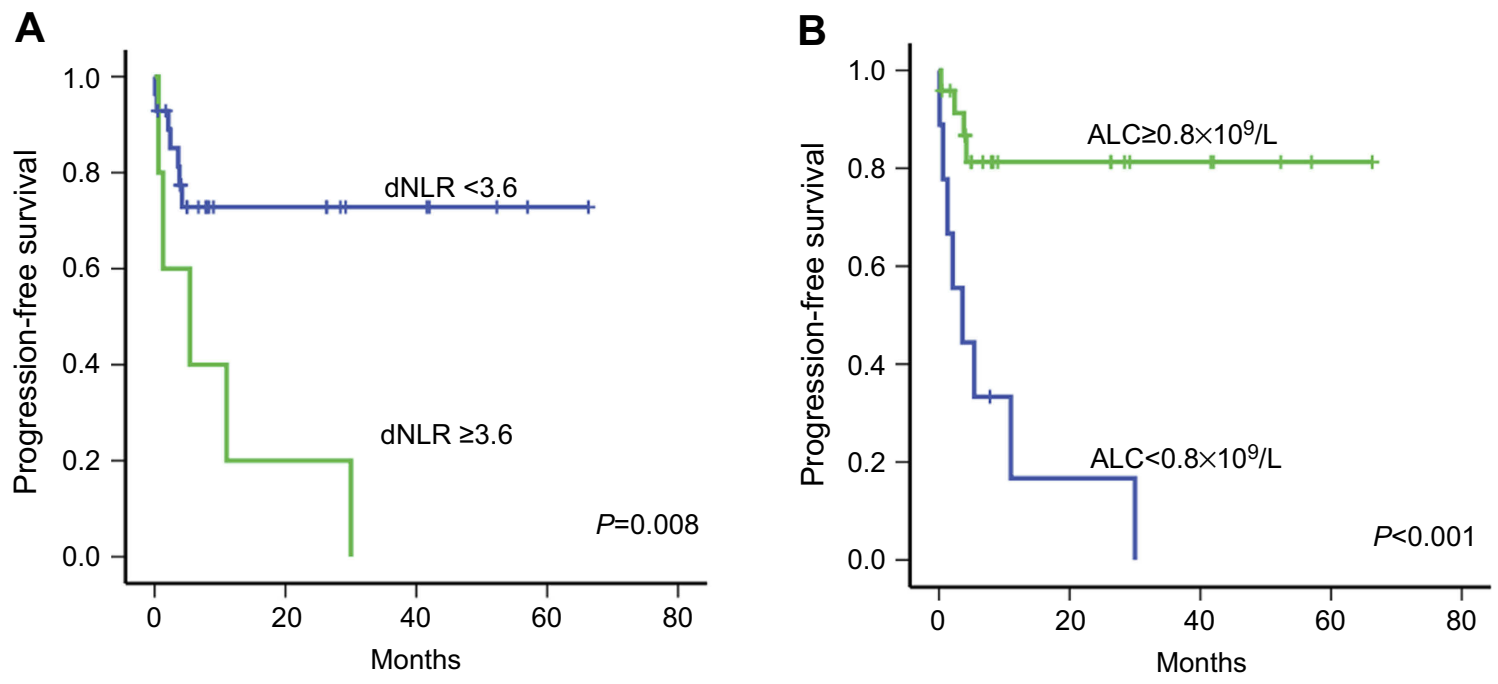

Figure 3 Kaplan-Meier curve of progression-free survival according to dNLR (A) and ALC (B) at diagnosis in patients with ENKTL.

Abbreviations: ALC, absolute lymphocyte count; AUC, area under the curve; dNLR, derived neutrophil to lymphocyte ratio; ENKTL, extranodal natural killer/T-cell lymphoma. 
A

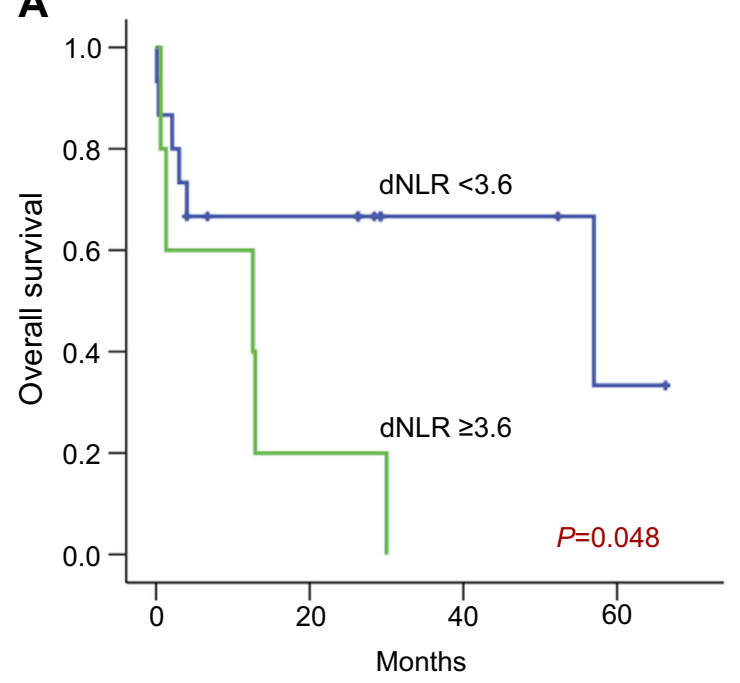

C

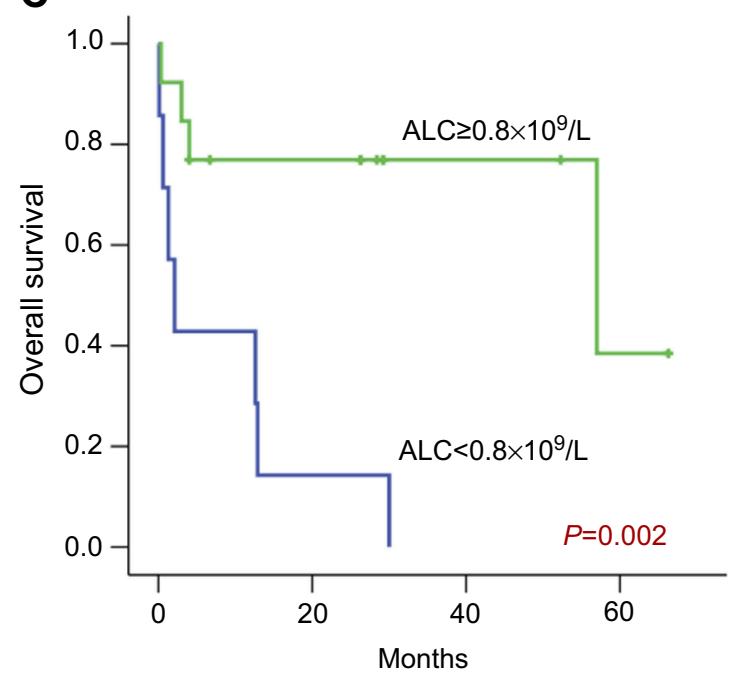

B

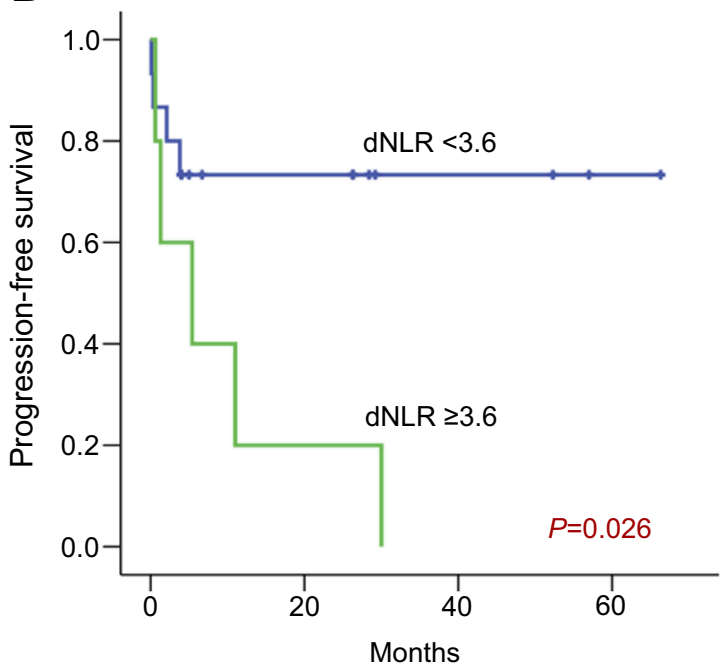

D

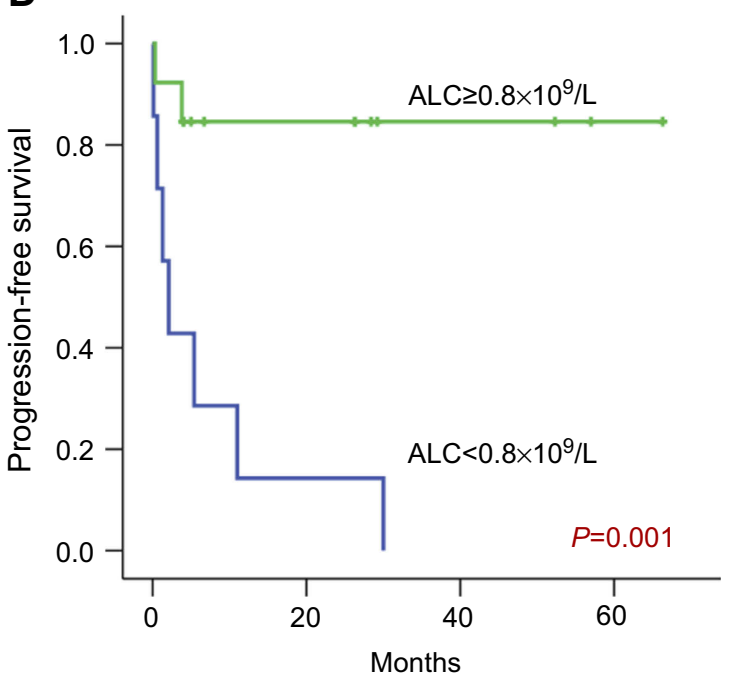

Figure 4 Kaplan-Meier curve of overall survival and progression-free survival according to dNLR (A and B) and ALC (C and D) at diagnosis in ENKTL patients with stage III/IV disease.

Abbreviations: ALC, absolute lymphocyte count; AUC, area under the curve; dNLR, derived neutrophil to lymphocyte ratio; ENKTL, extranodal natural killer/T-cell lymphoma.

with worse OS of ENKTL patients (Table 3). Next, we put the stage, ECOG PS score, serum LDH, distant lymphnode involvement, $\beta 2-\mathrm{MG}$, IPI score, ALC, and dNLR into the multivariate Cox model. In multivariate analysis, we identified that ALC $<0.8 \times 10^{9} / \mathrm{L}$ (HR, $36.023 ; 95 \% \mathrm{CI}$, 2.438-532.243; $P=0.009$ ) at diagnosis was an independent prognostic factor for the OS of ENKTL patients (Table 3). However, the dNLR value at diagnosis was not of significance in multivariate analysis.

In terms of PFS, univariable analysis identified several potential prognostic factors affecting PFS, including elevated serum LDH level (HR, 3.733; 95\%CI, 1.111-12.541; $P=0.033), \quad$ ALC $<0.8 \times 10^{9} / \mathrm{L} \quad(\mathrm{HR}, \quad 7.392 ; \quad 95 \% \mathrm{CI}$,
2.213-24.693; $P=0.001$ ), and dNLR $\geq 3.6$ (HR, 4.199; $95 \% \mathrm{CI}, 1.326-13.297 ; P=0.015)$. In multivariate Cox analysis, it was also found that $\mathrm{ALC}<0.8 \times 10^{9} / \mathrm{L}$ at diagnosis cloud independently predicts worse PFS in patients with ENKTL (HR, 7.698; 95\%CI, 1.573-37.679; $P=0.012$; Table 4).

\section{Discussion}

The host immune system, inflammation, and tumor microenvironment have been recognized to contribute to the development and progression of human malignancies. ${ }^{27}$ Lymphocyte count is a marker of host immune and lymphopenia is considered as a suggestion of immunocompetence. ${ }^{28}$ As 
Table 3 Univariate and multivariate Cox regression analysis of overall survival in patients with ENKTL

\begin{tabular}{|c|c|c|c|c|}
\hline \multirow[t]{3}{*}{ Prognostic factors } & \multicolumn{4}{|l|}{ Overall survival } \\
\hline & \multicolumn{2}{|l|}{ Univariate analysis } & \multicolumn{2}{|l|}{ Multivariate analysis } \\
\hline & HR $(95 \% \mathrm{Cl})$ & $P$-value & HR (95\% CI) & $P$-value \\
\hline Male sex & $0.342(0.095-1.227)$ & 0.1 & n.d. & n.d. \\
\hline Age $\geq 60$ years & $0.463(0.059-3.659)$ & 0.465 & n.d. & n.d. \\
\hline Stage III/IV & $7.378(0.949-57.353)$ & 0.056 & $9.202(0.567-149.365)$ & 0.119 \\
\hline B symptoms & $\mathrm{I} .285(0.38 \mathrm{I}-4.328)$ & 0.686 & n.d. & n.d. \\
\hline ECOG PS score $\geq 2$ & $4.865(1.477-16.030)$ & 0.009 & $2.520(0.183-34.801)$ & 0.490 \\
\hline Serum LDH > normal & $3.373(0.979-11.614)$ & 0.054 & $1.674(0.329-8.510)$ & 0.535 \\
\hline Distant lympho node involved & $3.237(0.705-14.857)$ & 0.131 & n.d. & n.d. \\
\hline BM involvement & $3.267(0.69 \mid-15.454)$ & 0.135 & n.d. & n.d. \\
\hline$\beta 2-M G \geq 2.8 \mathrm{mg} / \mathrm{L}$ & $4.795(1.223-18.809)$ & 0.025 & $0.556(0.030-10.246)$ & 0.693 \\
\hline Blood EBV DNA $\geq 5,000$ copies $/ \mathrm{mL}$ & $2.714(0.814-9.054)$ & 0.104 & n.d. & n.d. \\
\hline No response to treatment & $61.243(0.573-6,543.069)$ & 0.084 & n.d. & n.d. \\
\hline PINK score $\geq 2$ & $2.356(0.704-7.891)$ & 0.165 & n.d. & n.d. \\
\hline IPI Score $\geq 2$ & $6.332(1.379-29.074)$ & 0.018 & $1.358(0.142-12.955)$ & 0.790 \\
\hline $\mathrm{ALC}<0.8 \times 10^{9} / \mathrm{L}$ & $|1.25|(2.946-42.968)$ & 0.0004 & $36.023(2.438-532.243)$ & 0.009 \\
\hline$d N L R \geq 3.6$ & $5.938(1.790-19.704)$ & 0.004 & $0.103(0.05-2.098)$ & 0.140 \\
\hline
\end{tabular}

Note: Bold signifies $P<0.05$.

Abbreviations: ALC, absolute lymphocyte count; dNLR, derived neutrophil to lymphocyte ratio; ECOG PS, Eastern Cooperative Oncology Group performance status;

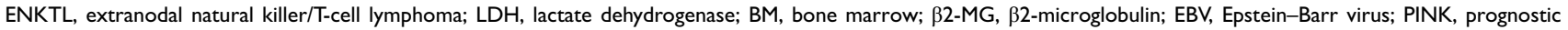
index for NK/T cell lymphoma; IPI, international Prognostic Index; ULN, upper limit of normal. n.d.: not done in multivariate analysis.

Table 4 Univariate and multivariate Cox regression analysis of progression-free survival in patients with ENKTL

\begin{tabular}{|c|c|c|c|c|}
\hline \multirow[t]{3}{*}{ Prognostic Factors } & \multicolumn{4}{|c|}{ Progression-free survival } \\
\hline & \multicolumn{2}{|l|}{ Univariate analysis } & \multicolumn{2}{|c|}{ Multivariate analysis } \\
\hline & HR $(95 \% \mathrm{Cl})$ & $P$-value & HR $(95 \% \mathrm{Cl})$ & $P$-value \\
\hline Male sex & $0.37 \mid(0.107-1.28 I)$ & 0.117 & n.d. & n.d. \\
\hline Age $\geq 60$ years & $1.664(0.439-6.312)$ & 0.454 & n.d. & n.d. \\
\hline Stage III/IV & $1.807(0.486-6.727)$ & 0.378 & n.d. & n.d. \\
\hline B symptoms & 1.351 (0.402-4.539) & 0.626 & n.d. & n.d. \\
\hline ECOG PS score $\geq 2$ & $2.568(0.809-8.147)$ & 0.109 & n.d. & n.d. \\
\hline Serum LDH > normal & $3.733(1 .|I|-\mid 2.54 I)$ & 0.033 & $2.063(0.534-7.968)$ & 0.294 \\
\hline Distant lympho node Involved & $4.412(0.963-20.206)$ & 0.056 & n.d. & n.d. \\
\hline BM involvement & $2.799(0.598-13.098)$ & 0.191 & n.d. & n.d. \\
\hline$\beta 2-M G \geq 2.8 \mathrm{mg} / \mathrm{L}$ & $3.350(0.972-|| .54 \mid)$ & 0.055 & n.d. & n.d. \\
\hline Blood EBV DNA $\geq 5,000$ copies $/ \mathrm{mL}$ & $3.588(0.968-13.295)$ & 0.056 & n.d. & n.d. \\
\hline No response to treatment & $68.634(0.655-7,191.306)$ & 0.075 & n.d. & n.d. \\
\hline PINK score $\geq 2$ & $\mathrm{I} .405(0.442-4.47 \mathrm{I})$ & 0.564 & n.d. & n.d. \\
\hline IPI Score $\geq 2$ & $3.426(0.923-12.717)$ & 0.066 & n.d. & n.d. \\
\hline ALC $<0.8 \times 10^{9} / \mathrm{L}$ & $7.392(2.213-24.693)$ & 0.001 & $7.698(1.573-37.679)$ & 0.012 \\
\hline $\mathrm{dNLR} \geq 3.6$ & $4.199(1.326-13.297)$ & 0.015 & $0.615(0.131-2.894)$ & 0.539 \\
\hline
\end{tabular}

Note: Bold signifies $P<0.05$.

Abbreviations: ALC, absolute lymphocyte count; dNLR, derived neutrophil to lymphocyte ratio; ECOG PS, Eastern Cooperative Oncology Group performance status;

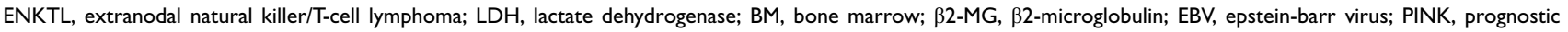
index for NK/T cell lymphoma; IPI, international prognostic index; ULN, upper limit of normal. n.d.: not done in multivariate analysis.

a surrogate marker of host immunity, ALC has a potent effect in anti-tumor immunity, and is related to the treatment response of several hematological neoplasms. It was reported that ALC higher than 350 cells $/ \mu \mathrm{L}$ measured on the 33rd day of induction therapy was associated with better OS and eventfree survival (EFS) in acute lymphoblastic leukemia (ALL). ${ }^{29}$ 
Moreover, the day $100 \mathrm{ALC} / \mathrm{AMC}$ ratio could independently predict the outcomes in children, adolescents, and young adults with refractory/relapsed (R/R) HL who underwent autologous stem cell transplantation (ASCT) ${ }^{24} \mathrm{Kim}$ et al illustrated that an early recovery of ALC $\left(>0.4 \times 10^{9} / \mathrm{L}\right.$ at 15 days) after frontline ASCT can be regarded as a good prognostic marker in patients diffuse large B-cell lymphoma (DLBCL) ${ }^{30}$ In this study, we identified that ENKTL patients with low ALC $\left(<0.8 \times 10^{9} / \mathrm{L}\right)$ revealed inferior response to treatment and shorter survival time compared to those with higher $\operatorname{ALC}\left(\geq 0.8 \times 10^{9} / \mathrm{L}\right)$.

Previous studies mainly focused on the involvement and prognostic value of non-immune factors in ENKTL, such as EBV DNA. ${ }^{31}$ More recently, several studies investigated the prognostic value of ALC in T-cell NHL. Peripheral T-cell lymphomas - not otherwise specified (PTCL-NOS) patients with lower ALC/AMC ratios showed shorter survival than patients with high ALC/AMC ratios. ${ }^{32}$ Our study retrospectively evaluated the prognostic significance of ALC in 33 newly diagnosed ENKTL patients. Through univariate analysis as well as multivariate analysis, we identified that low ALC is a poor independent prognostic factor for clinical outcome. Consistent with previous studies, the decreasing ALC, a circulating immune cell which was considered as an indicator of immune suppression, was considered as an unfavorable prognostic marker for ENKTL. ${ }^{33}$

The peripheral pro-inflammatory parameters, such as neutrophils, albumin, LDH, and C-reactive protein (CRP), have been reported to be associated with poor outcomes in patients with tumors. ${ }^{34}$ As an indicator of systemic inflammation, the dNLR was validated to act as an independent prognostic factor in cancer. ${ }^{26}$ The dNLR has been proposed as an alternative to NLR because of its convenience in cases with only WBC and ANC recorded. ${ }^{26}$ It was demonstrated that high dNLR was associated with poor survival outcome in a variety of tumor types, including melanoma, pancreatic cancer, bladder cancer, and renal cancer. ${ }^{35-39}$ As it is inexpensive and easy to calculate, dNLR could be routinely detected in the clinical practice. Recently, several studies focused on the prognostic value of dNLR in patients receiving immuno-modulating therapy. In melanoma, dNLR of $\geq 3$ exhibited an independent negative effect on the OS of ipilimumab-treated melanoma patients $(n=720) .{ }^{35}$ In addition, the pretreatment dNLR level in NSCLC was correlated with poor clinical outcome for checkpoint inhibitor therapy. ${ }^{10}$ In hematological malignancies, Troppan et al reported that $\mathrm{dNLR} \geq 1.8$ was a significant factor for 5-year disease-free survival (DFS) in DLBCL patients treated with standard rituximab and CHOP (R-CHOP) regimen. ${ }^{15}$ Elevated NLR $(\geq 2.95)$ was related to inferior OS in MM patients receiving induction therapy with bortezomib-based regimens. ${ }^{40}$ Our present study demonstrated that ENKTL patients who received first-line therapy with high dNLR $(\geq 3.6)$ presented lower response (CR or PR) rate when compared to patients with lower dNLR.

The prognostic effects of dNLR in hematological malignancies have been recently reported. Elevated dNLR level was an independent poor prognostic factor for transplantation-ineligible $\mathrm{MM}$ patients with $\mathrm{MM}^{41}$ Independent significant association between dNLR and poor OS as well as DFS was identified in DLBCL. ${ }^{15}$ However, the prognostic significance of dNLR in ENKTL has not been reported. By multivariate analysis, we found that dNLR levels were associated with poor OS and PFS in ENKTL patients. Nevertheless, no significant effect was found in dNLR in multivariable analysis, which might be related to the small number of included cases and relatively short time of follow-up.

However, there are still several limitations to this study. This is a retrospective study in a single center. Prospective randomized studies are warranted to verify the significance of these indexes for patients after firstline therapy. Moreover, due to the rarity of ENKTL, the number of patients included in this study is limited. Further investigations with larger population cohorts and longer median follow-up are needed to validate the prognostic value of dNLR, ALC in ENKTL patients.

\section{Conclusion}

In conclusion, our finding demonstrated that elevated dNLR ( $\geq 3.6)$ was associated with short survival time and inferior therapeutic response in ENKTL, which may act as a potential prognostic factor in patients with ENKTL. Low $\operatorname{ALC}\left(<0.8 \times 10^{9} / \mathrm{L}\right)$ at diagnosis of ENKTL represented an independent poor prognostic factor for clinical outcome. The dNLR and ALC may serve as a novel, convenient, inexpensive and independent prognostic factors for patients with untreated ENKTL.

\section{Acknowledgments}

This study was partly supported by National Natural Science Foundation (No. 81800194, No. 81770210, No. 81600121, No. 81473486), Natural Science Foundations of Shandong Province (Nos. ZR2018BH011, ZR2016HQ46), Technology 
Development Projects of Shandong Province (No. 2017GSF18189, No. 2016GSF201029), Key Research and Development Program of Shandong Province (No. 2018CXGC1213), Medical and Health Technology Innovation Program of Jinan City (No. 201805065), Program of Shandong Medical Leading Talent, and Taishan Scholar Foundation of Shandong Province.

\section{Disclosure}

The authors report no conflicts of interest in this work.

\section{References}

1. Haverkos BM, Pan Z, Gru AA, et al. Extranodal NK/T Cell Lymphoma, Nasal Type (ENKTL-NT): an update on epidemiology, clinical presentation, and natural history in North American and European cases. Curr Hematol Malig Rep. 2016;11(6):514-527. doi:10.1007/s11899-016-0355-9

2. Vose J, Armitage J, Weisenburger D. International peripheral T-cell and natural killer/T-cell lymphoma study: pathology findings and clinical outcomes. J Clin Oncol. 2008;26(25):4124 4130. doi:10.1200/ JCO.2008.16.4558

3. Kwong YL, Kim WS, Lim ST, et al. SMILE for natural killer/T-cell lymphoma: analysis of safety and efficacy from the Asia Lymphoma Study Group. Blood. 2012;120(15):2973-2980. doi:10.1182/blood2012-05-431460

4. Au WY, Weisenburger DD, Intragumtornchai T, et al. Clinical differences between nasal and extranasal natural killer/T-cell lymphoma: a study of 136 cases from the international peripheral T-Cell lymphoma project. Blood. 2009;113(17):3931-3937. doi:10.1182/blood-2008-10-185256

5. Tse E, Kwong YL. The diagnosis and management of NK/T-cell lymphomas. J Hematol Oncol. 2017;10(1):85. doi:10.1186/s13045017-0452-9

6. Yamaguchi M, Suzuki R, Oguchi M. Advances in the treatment of extranodal NK/T-cell lymphoma, nasal type. Blood. 2018;131 (23):2528-2540. doi:10.1182/blood-2017-12-791418

7. Yamaguchi M, Kwong YL, Kim WS, et al. Phase II study of SMILE chemotherapy for newly diagnosed stage IV, relapsed, or refractory extranodal natural killer (NK)/T-cell lymphoma, nasal type: the NK-Cell Tumor Study Group study. J Clin Oncol. 2011;29 (33):4410-4416. doi:10.1200/JCO.2011.35.6287

8. International Non-Hodgkin's Lymphoma Prognostic Factors Project. A predictive model for aggressive non-Hodgkin's lymphoma. N Engl J Med. 1993;329(14):987-994.

9. Kim SJ, Yoon DH, Jaccard A, et al. A prognostic index for natural killer cell lymphoma after non-anthracycline-based treatment: a multicentre, retrospective analysis. Lancet Oncol. 2016;17 (3):389-400. doi:10.1016/S1470-2045(15)00533-1

10. Mezquita L, Auclin E, Ferrara R, et al. Association of the lung immune prognostic index with immune checkpoint inhibitor outcomes in patients with advanced non-small cell lung cancer. JAMA Oncol. 2018;4(3):351-357. doi:10.1001/jamaoncol.2017.4771

11. Capone M, Giannarelli D, Mallardo D, et al. Baseline neutrophil-tolymphocyte ratio (NLR) and derived NLR could predict overall survival in patients with advanced melanoma treated with nivolumab. $J$ Immunother Cancer. 2018;6(1):74. doi:10.1186/ s40425-018-0383-1

12. Cox S, Hurt C, Grenader T, Mukherjee S, Bridgewater J, Crosby T. The prognostic value of derived neutrophil to lymphocyte ratio in oesophageal cancer treated with definitive chemoradiotherapy. Radiother Oncol. 2017;125(1):154-159. doi:10.1016/j.radonc.2017. 08.023
13. Song S, Li C, Li S, Gao H, Lan X, Xue Y. Derived neutrophil to lymphocyte ratio and monocyte to lymphocyte ratio may be better biomarkers for predicting overall survival of patients with advanced gastric cancer. Onco Targets Ther. 2017;10:3145-3154. doi:10.2147/ OTT.S138039

14. Templeton AJ, Rodriguez-Lescure A, Ruiz A, et al. Prognostic role for the derived neutrophil-to-lymphocyte ratio in early breast cancer: a GEICAM/9906 substudy. Clin Transl Oncol. 2018;20(12):15481556. doi:10.1007/s12094-018-1885-5

15. Troppan K, Deutsch A, Gerger A, et al. The derived neutrophil to lymphocyte ratio is an independent prognostic factor in patients with diffuse large B-cell lymphoma. Br J Cancer. 2014;110(2):369-374. doi:10.1038/bjc.2013.763

16. Quigley DA, Kristensen V. Predicting prognosis and therapeutic response from interactions between lymphocytes and tumor cells. Mol Oncol. 2015;9(10):2054-2062. doi:10.1016/j.molonc.2015.10.003

17. Feng F, Zheng G, Wang Q, et al. Low lymphocyte count and high monocyte count predicts poor prognosis of gastric cancer. $B M C$ Gastroenterol. 2018;18(1):148. doi:10.1186/s12876-018-0863-2

18. Joseph N, Dovedi SJ, Thompson C, et al. Pre-treatment lymphocytopaenia is an adverse prognostic biomarker in muscle-invasive and advanced bladder cancer. Ann Oncol. 2016;27(2):294-299. doi:10.1093/annonc/mdv546

19. Milne K, Alexander C, Webb JR, et al. Absolute lymphocyte count is associated with survival in ovarian cancer independent of tumor-infiltrating lymphocytes. J Transl Med. 2012;10:33. doi:10.1186/ 1479-5876-10-233

20. Suriu C, Akria L, Azoulay D, Shaoul E, Barhoum M, Braester A. Absolute lymphocyte count as a prognostic marker in newly diagnosed multiple myeloma patients. Int J Lab Hematol. 2016;38(3): e56-e59. doi:10.1111/ijlh.12482

21. Jang JE, Kim YR, Kim SJ, et al. A new prognostic model using absolute lymphocyte count in patients with primary central nervous system lymphoma. Eur J Cancer. 2016;57:127-135. doi:10.1016/j. ejca.2016.01.016

22. Jia T, Zhang R, Zhu HY, et al. Prognostic significance of peripheral blood absolute monocyte count and lymphocyte to monocyte ratio in anaplastic large cell lymphoma. Cancer Biomark. 2018;22 (4):807-813. doi:10.3233/CBM-181505

23. Hasenclever D, Diehl V. A prognostic score for advanced hodgkin's disease. International prognostic factors project on advanced hodgkin's disease. N Engl J Med. 1998;339(21):1506-1514. doi:10.1056/ NEJM199811193392104

24. Galvez-Silva J, Maher OM, Park M, et al. Prognostic analysis of absolute lymphocyte and monocyte counts after autologous stem cell transplantation in children, adolescents, and young adults with refractory or relapsed Hodgkin lymphoma. Biol Blood Marrow Transplant. 2017;23(8):1276-1281. doi:10.1016/j.bbmt.2017.04.013

25. Swerdlow SH, Campo E, Pileri SA, et al. The 2016 revision of the World Health Organization classification of lymphoid neoplasms. Blood. 2016;127(20):2375-2390. doi:10.1182/blood-2016-01-643569

26. Proctor MJ, McMillan DC, Morrison DS, Fletcher CD, Horgan PG, Clarke SJ. A derived neutrophil to lymphocyte ratio predicts survival in patients with cancer. Br J Cancer. 2012;107(4):695-699. doi:10.1038/bjc.2012.292

27. Diakos CI, Charles KA, McMillan DC, Clarke SJ. Cancer-related inflammation and treatment effectiveness. Lancet Oncol. 2014;15 (11):e493-e503. doi:10.1016/S1470-2045(14)70263-3

28. Jacobson CA, LaCasce AS. Lymphoma: risk and response after solid organ transplant. Oncology (Williston Park). 2010;24 (10):936-944.

29. Farkas T, Muller J, Dj E, Csoka M, Gt K. Absolute Lymphocyte Count (ALC) after induction treatment predicts survival of pediatric patients with acute lymphoblastic leukemia. Pathol Oncol Res. 2017;23(4):889-897. doi:10.1007/s12253-017-0 192-8 
30. Kim Y, Kim SJ, Cheong JW, et al. Clinical impact of early recovery of peripheral blood absolute lymphocyte count after frontline autologous stem cell transplantation for diffuse large B-cell lymphoma. Hematol Oncol. 2017;35(4):465-471. doi:10.1002/ hon. 2332

31. Chen R, Wang C, Zhou Y, Wen B. Prognostic implications of circulating Epstein-Barr virus DNA for extranodal natural killer/T-cell lymphoma, nasal type: a meta-analysis. Cancer Manag Res. 2018;10:2183-2192. doi:10.2147/CMAR.S162168

32. Li Q, Gao S, Ma J, et al. A lower ALC/AMC ratio is associated with poor prognosis of peripheral T-cell lymphoma-not otherwise specified. Leuk Res. 2018;73:5-11. doi:10.1016/j.leukres.2018.07.020

33. Huang JJ, Jiang WQ, Lin TY, et al. Absolute lymphocyte count is a novel prognostic indicator in extranodal natural killer/T-cell lymphoma, nasal type. Ann Oncol. 2011;22(1):149-155. doi:10.1093/ annonc/mdq314

34. Laird BJ, Fallon M, Hjermstad MJ, et al. Quality of life in patients with advanced cancer: differential association with performance status and systemic inflammatory response. J Clin Oncol. 2016;34(23):2769-2775. doi:10.1200/JCO.2015.65.7742

35. Ferrucci PF, Ascierto PA, Pigozzo J, et al. Baseline neutrophils and derived neutrophil-to-lymphocyte ratio: prognostic relevance in metastatic melanoma patients receiving ipilimumab. Ann Oncol. 2016;27(4):732-738. doi:10.1093/annonc/mdw016
36. Suzuki R, Takagi T, Hikichi T, et al. Derived neutrophil/lymphocyte ratio predicts gemcitabine therapy outcome in unresectable pancreatic cancer. Oncol Lett. 2016;11(5):3441-3445. doi:10.3892/ol.2016.4381

37. van Kessel KE, de Haan LM, Fransen van de Putte EE, et al. Elevated derived neutrophil-to-lymphocyte ratio corresponds with poor outcome in patients undergoing pre-operative chemotherapy in muscle-invasive bladder cancer. Bladder Cancer. 2016;2(3):351-360. doi:10.3233/BLC-160055

38. Amato RJ, Flaherty A, Zhang Y, Ouyang F, Mohlere V. Clinical prognostic factors associated with outcome in patients with renal cell cancer with prior tyrosine kinase inhibitors or immunotherapy treated with everolimus. Urol Oncol. 2014;32(3):345-354. doi:10.1016/j.urolonc.2013.09.008

39. Grenader T, Nash S, Plotkin Y, et al. Derived neutrophil lymphocyte ratio may predict benefit from cisplatin in the advanced biliary cancer: the ABC-02 and BT-22 studies. Ann Oncol. 2015;26 (9):1910-1916. doi:10.1093/annonc/mdv253

40. Zhou X, Wang J, Xia J, et al. Evaluation of neutrophil-to-lymphocyte ratio in newly diagnosed patients receiving borte- zomib-based therapy for multiple myeloma. Cancer Biomark. 2018;22(1):43-48. doi:10.3233/CBM-170795

41. Uz B. The prognostic value of the derived neutrophil-to-lymphocyte ratio in transplantation-ineligible patients with multiple myeloma. Acta Haematol. 2018;140(3):157-158. doi:10.1159/000491988

\section{Publish your work in this journal}

Cancer Management and Research is an international, peer-reviewed open access journal focusing on cancer research and the optimal use of preventative and integrated treatment interventions to achieve improved outcomes, enhanced survival and quality of life for the cancer patient.
The manuscript management system is completely online and includes a very quick and fair peer-review system, which is all easy to use Visit http://www.dovepress.com/testimonials.php to read real quotes from published authors. 